تاثير اسيدلينولئيك كنزوَّه بر بيان زن هاي هدف دركير در سوخت و ساز جربى

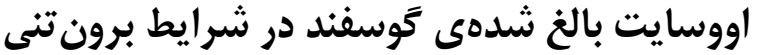

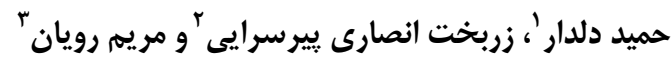

ا- دانشيار كروه علوم دامى، دانشكده علوم دامى و شيلات، دانشكاه علوم كشاورزى و منابع طبيعى سارى، (نويسنده مسوول:

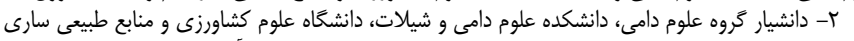

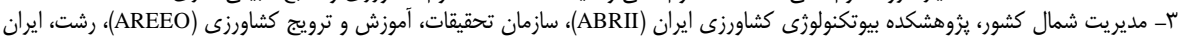
تاريخ يذيرش:

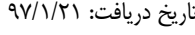

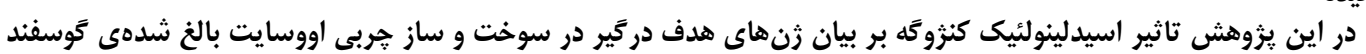

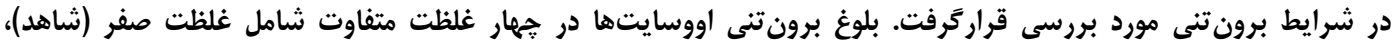

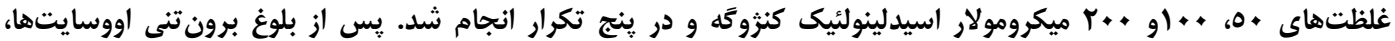

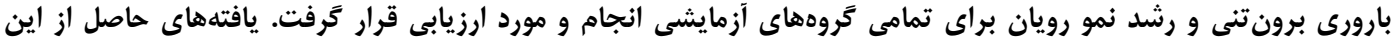

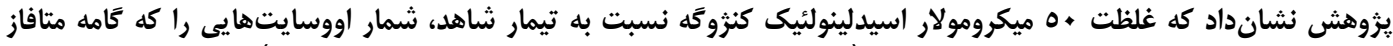

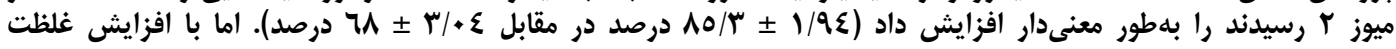

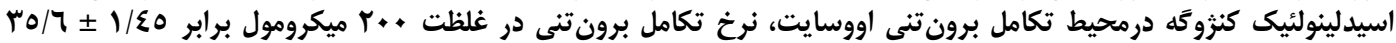

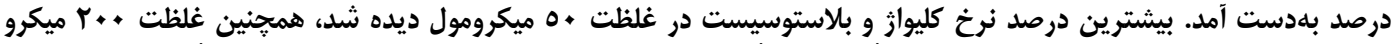

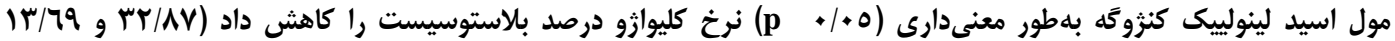

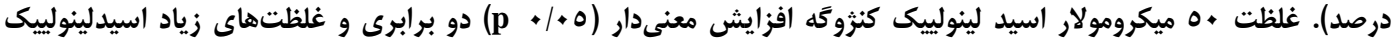

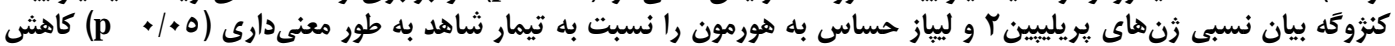

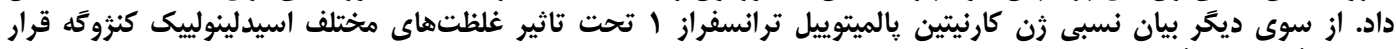

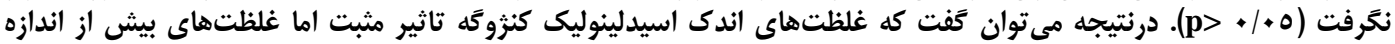

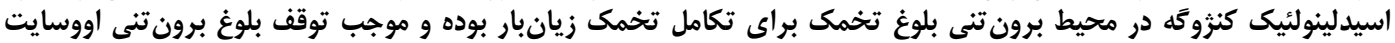

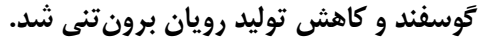

وازههاى كليدى: اسيدلينوليكى كنزوته، بلوغ برون تنى اووسايت، رشد و نمو رويان، سوخت و ساز هربى

تاثيرات به خصوص در اسيدجرب غيراشباع نوع امكآ و امكاو

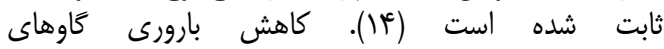

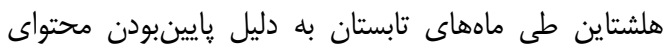

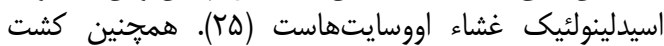

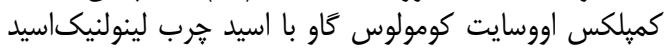

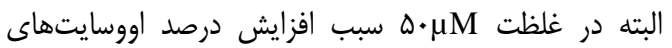

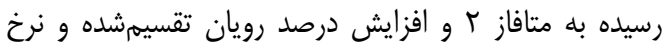

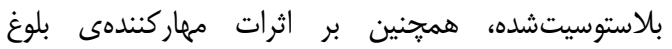

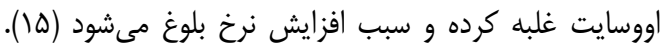

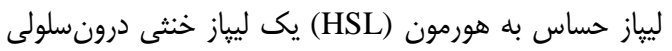

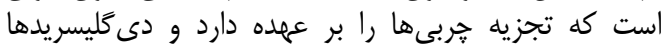

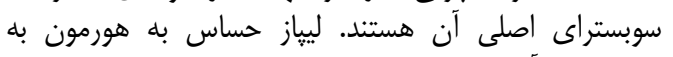

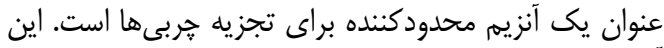

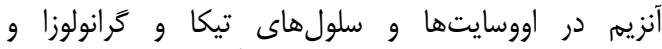

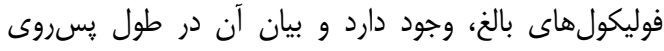

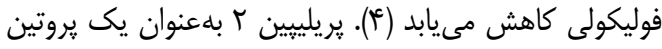

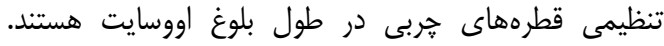

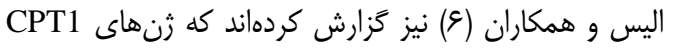

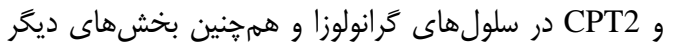

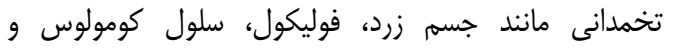
اووسايت نابالغ كاو بيان شده است. اين مطالعات نشان مىدهد

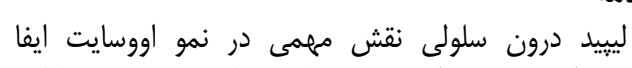

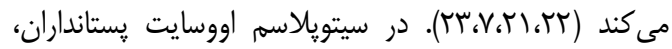

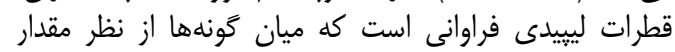

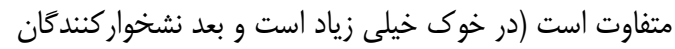

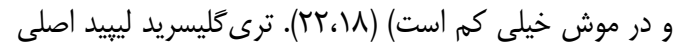

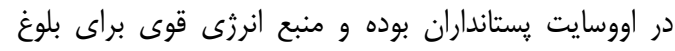

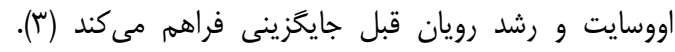

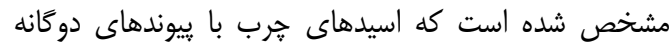

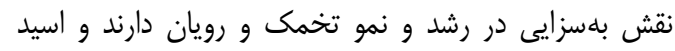

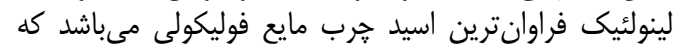

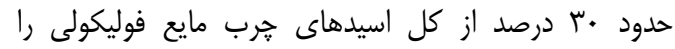

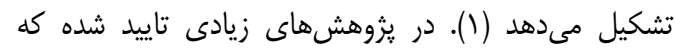

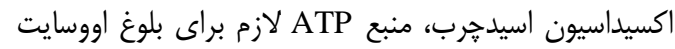

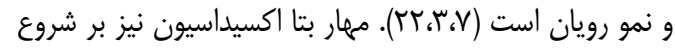

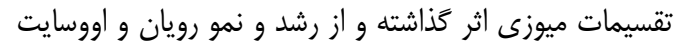

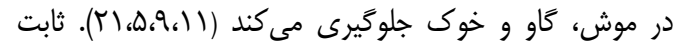

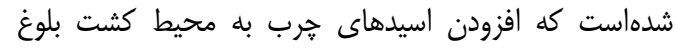

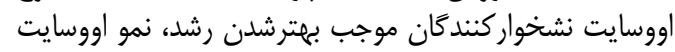

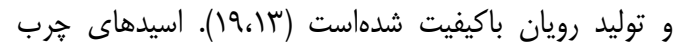
تاثير باسزايى بر افزايش عملكرد توليد مثلى دارند و ايناين 
ساخته شد و براى بيان نسبى ثنهاى موردنظر تا زمان انجام

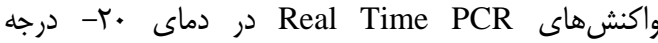

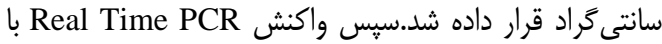

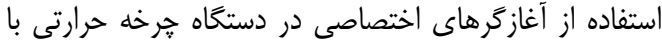

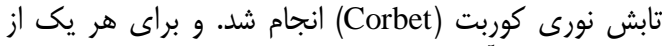
Real Time PCR نمونهها جرخه آستانه تعيين شد. بيان ثن

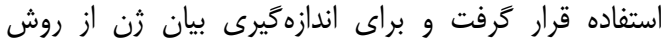

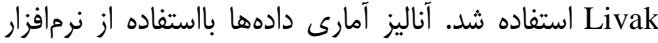
انجام شد. براى تجزيه دادهها از رويه GLMS

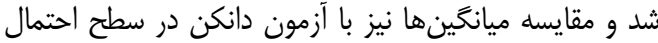

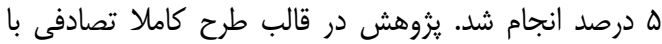

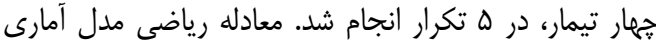

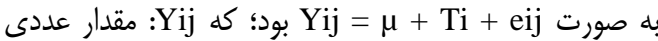

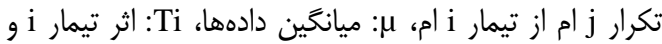

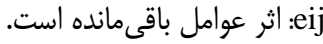

\section{نتايج و بحث}

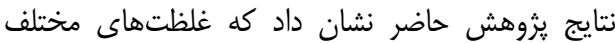

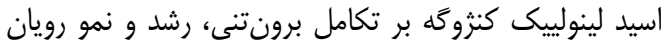

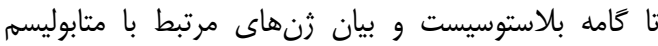

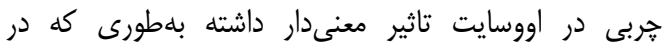

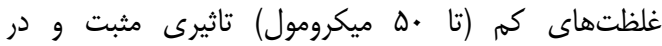

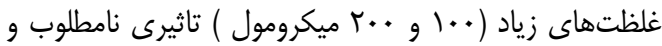

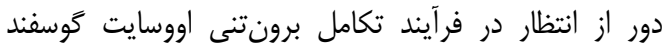

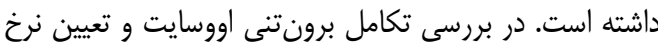

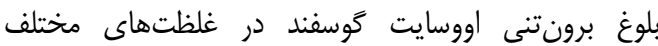

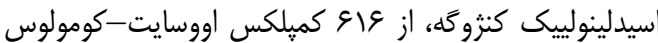

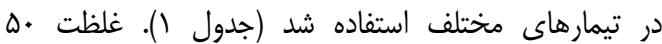

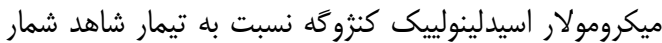

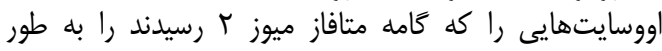

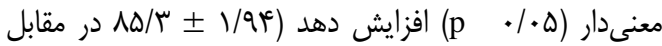

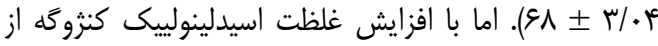

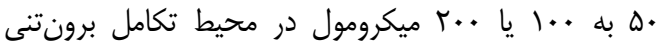

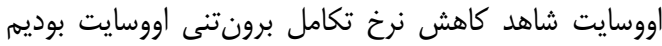

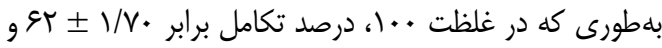

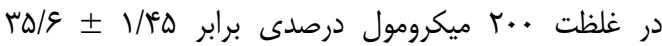

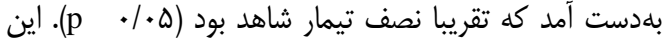

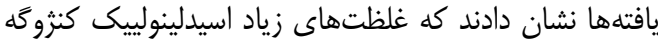

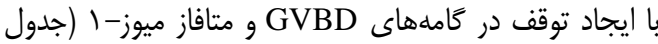

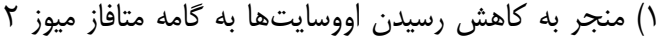

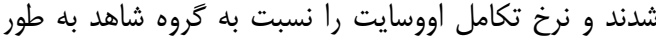

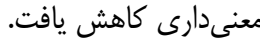

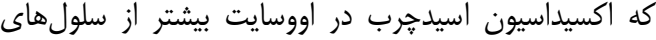

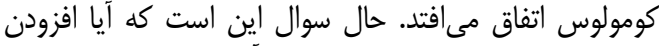

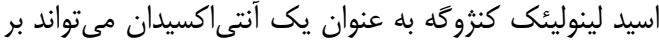

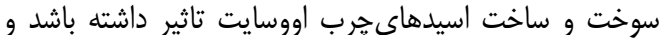

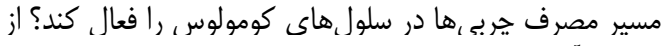

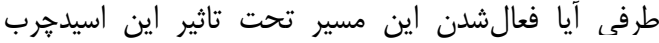

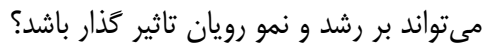

\section{مواد و روشها}

براى تهيهى كميلكس اووسايت كومولوس، تخمدان

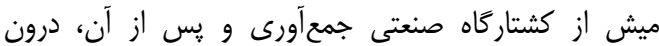

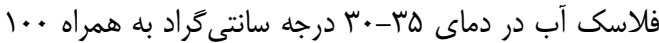

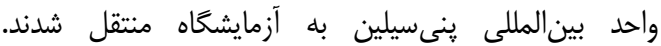

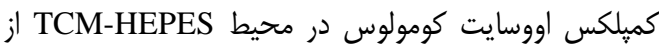

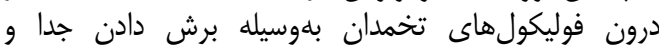

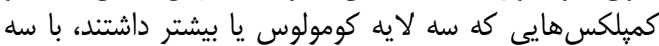

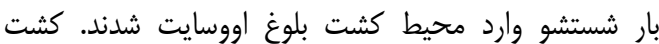

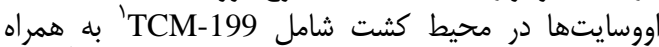

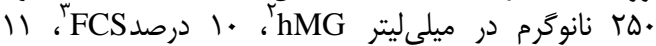

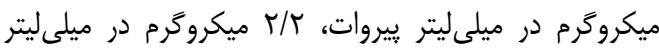

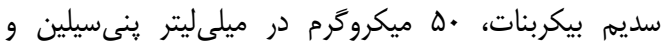

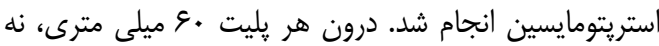

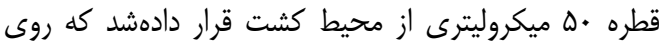

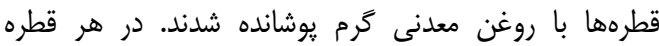

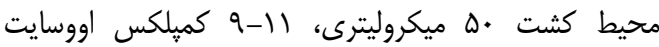

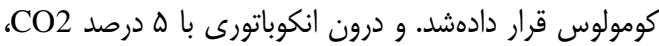

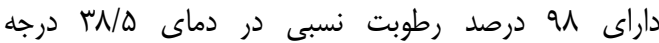
سانتى

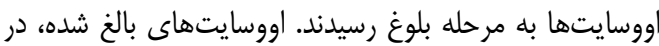

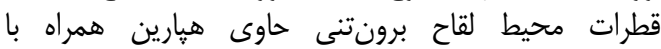

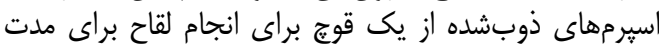

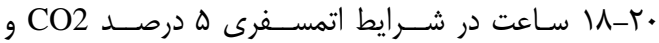

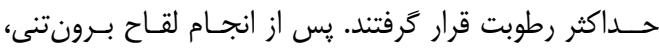

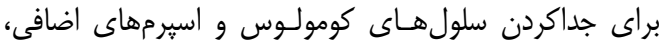

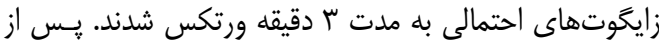

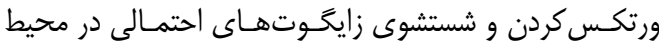

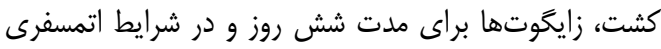

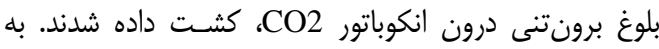

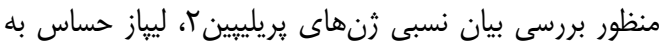

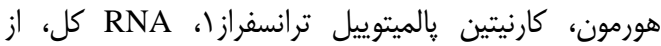

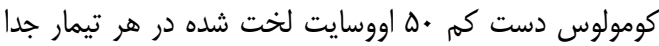

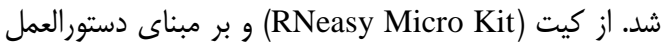

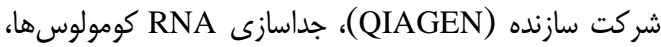

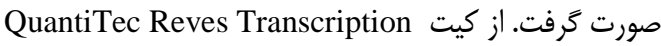
و براساس ثروتكل مربوطه، QIAGEN, 205311) 
جدول ا- تكامل برون تنى اووسايتهاى گَوسفند در غلظتهاى مختلف اسيد لينولييك كنثوگَه Table 1. Maturation of sheep oocyte in diferent concentrations of conjugated linoleic acid

\begin{tabular}{|c|c|c|c|c|c|}
\hline \multicolumn{4}{|c|}{ كامههاى مختلف تقسيم ميتوز (درصد) } & \multirow{2}{*}{ شمار اووسايت } & \multirow{2}{*}{ 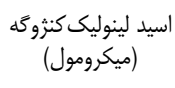 } \\
\hline MII & MI & GVBD & GV & & \\
\hline $9 \wedge \pm \Psi / \cdot \kappa^{b}$ & $19 \pm \cdot / \lambda V^{c}$ & $\mid r / q \pm \cdot / \Lambda V^{b}$ & $r / r \pm \cdot / r F^{\mathrm{a}}$ & 148 & صفر \\
\hline$\Lambda \omega / r \pm 1 / q \varphi^{\mathrm{a}}$ & $\Lambda \pm \cdot / \Delta r^{d}$ & $r \pm \cdot / \mu^{d}$ & $r / q \pm \cdot / \mu^{a}$ & IQT & $\Delta$. \\
\hline$g r \pm I / r .^{b}$ & $r r / r \pm \cdot / q r^{b}$ & $9 \pm \cdot / 4 \cdot{ }^{c}$ & $\Delta / \& \pm \cdot / \kappa T^{a}$ & 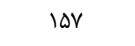 & $1 .$. \\
\hline 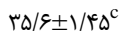 & $f \mid \pm \cdot / \mu \varphi^{\mathrm{a}}$ & $r \mid q \pm \cdot / \mu^{a}$ & $11 / 9 \pm \cdot / \varphi^{6} \varphi^{b}$ & 191 & $r \cdot$. \\
\hline
\end{tabular}

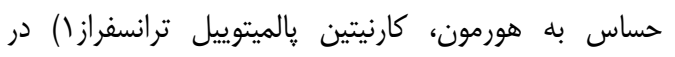

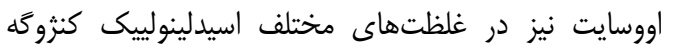

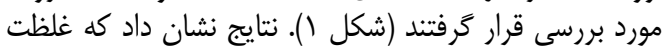

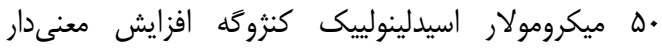

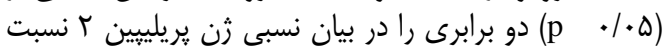

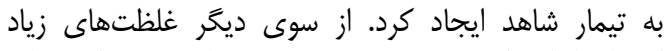

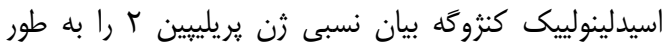

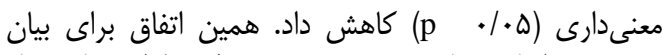

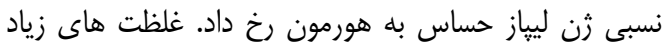

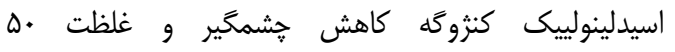

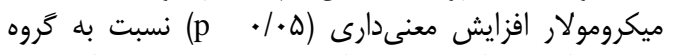

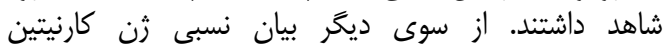

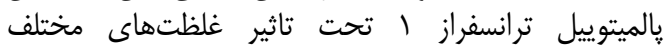

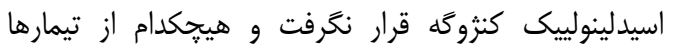

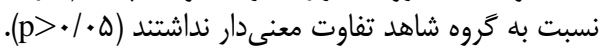

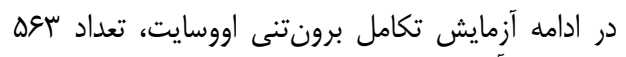

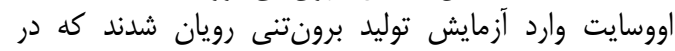

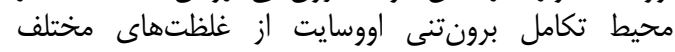

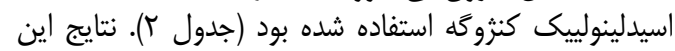

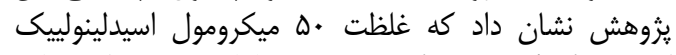

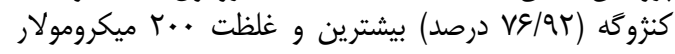

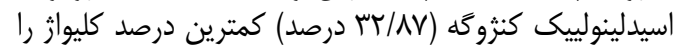

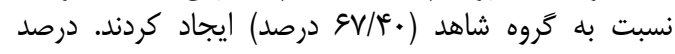

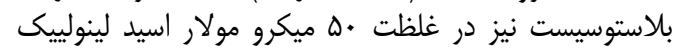

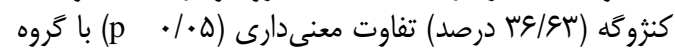

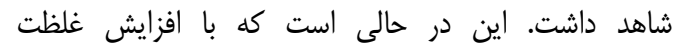

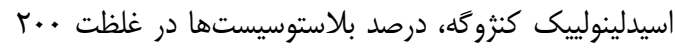

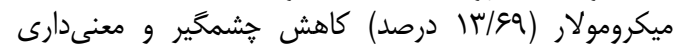

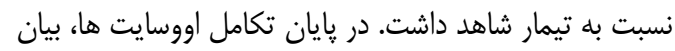

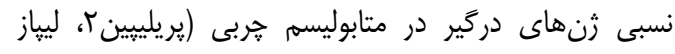

جدول r- نرخ رويان برونتنى گوسفند در غلظتهاى مختلف اسيد لينولييك كنثوكه Table 2. In vitro embryonic development of sheep oocytes in diferent concentrations of conjugated linoleic acid

\begin{tabular}{|c|c|c|c|}
\hline درصد بلاستوسيست (تعداد) & درصد كليواز (تعداد) & شمار اووسايت & اسيد لينوليك كنثوكه (ميكرومول) \\
\hline$\left(f^{c} \cdot\right)^{r q / q} r^{b}$ & (१) $q V / F^{\circ} \cdot{ }^{b}$ & ind & صفر \\
\hline 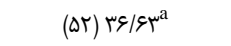 & $(11). v \varepsilon / a r^{a}$ & TFi & $\Delta$. \\
\hline 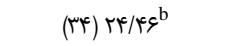 & $(V \Delta) \Delta r / Q \Delta^{c}$ & irq & $1 \ldots$ \\
\hline$(r \cdot) \mid r / 9 q^{c}$ & $(F \wedge) r r / A V^{c}$ & ifs & $r+$. \\
\hline
\end{tabular}



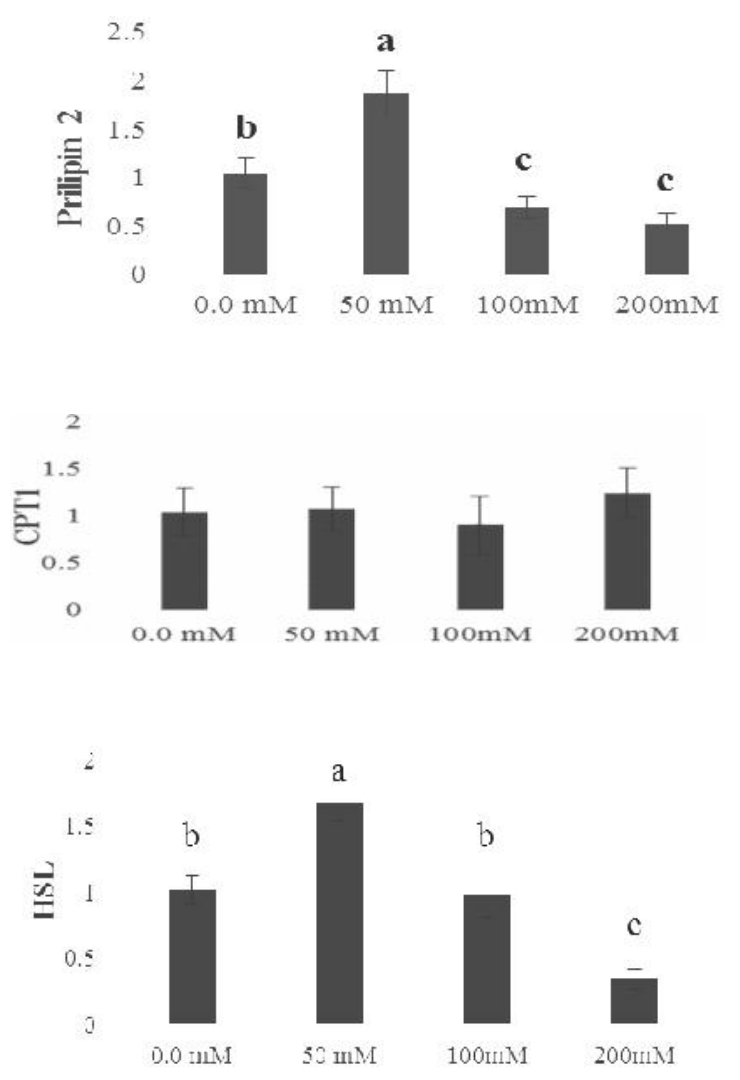

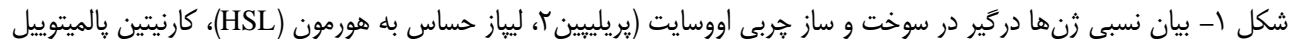

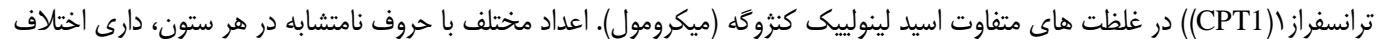

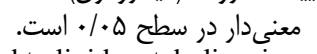

Figure 1. The relative gene expressions related to lipid metabolism in oocyte in diferent concentrations of conjugated linoleic acid.

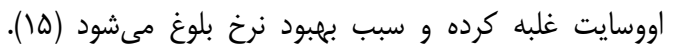

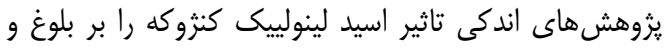

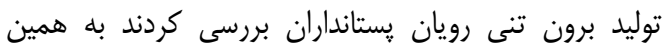

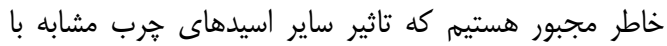

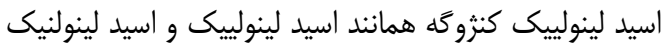

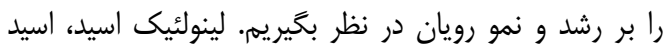

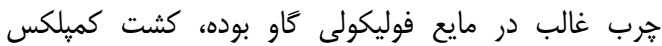

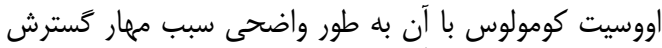

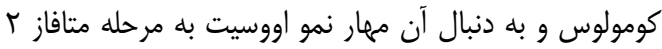

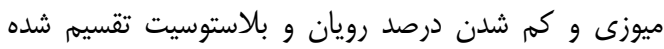

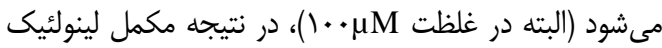

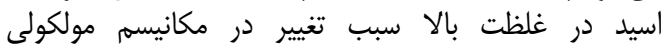

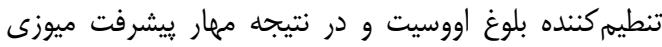

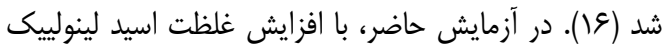

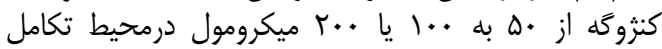

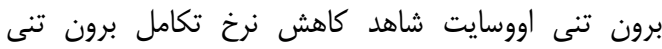

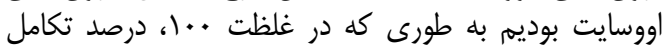

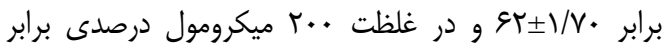

نتايج يزوهش حاضر نشان داد كه غلظتهاى مختلفي

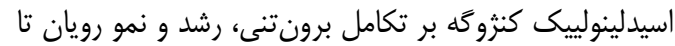

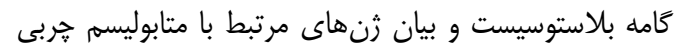

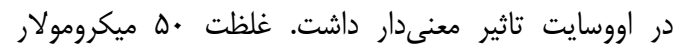

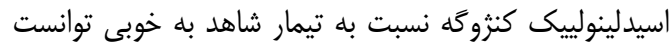

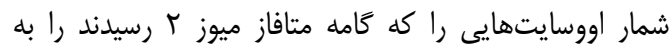

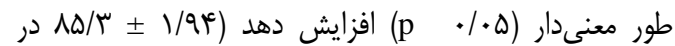

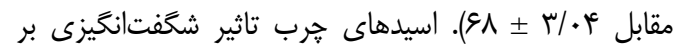

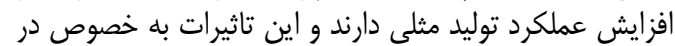

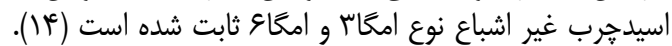

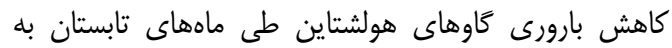

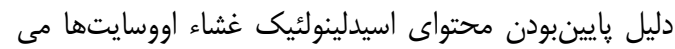

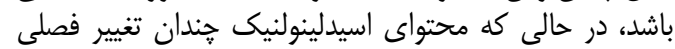

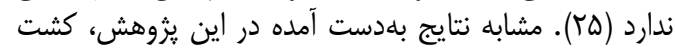

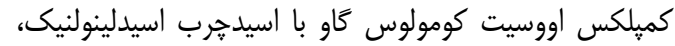

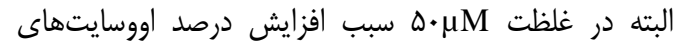

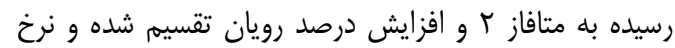

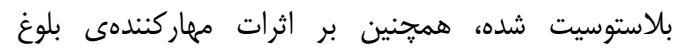


يافتههاى بلهدست آمده از يزوهش حاضر نشان داد كه

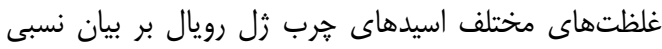

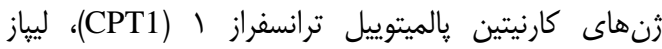

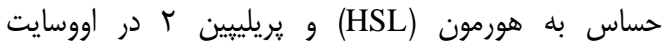

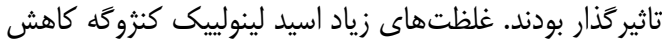

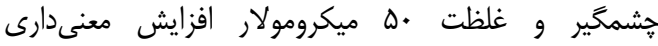
نسبت به گروه شاهد داشتند. در راستاى نتايج

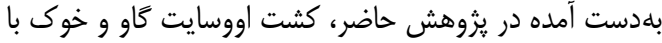

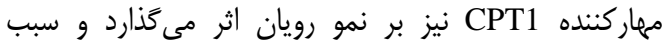

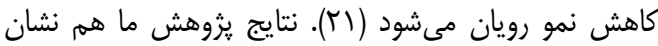

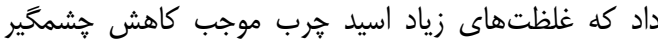

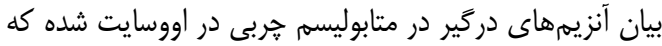

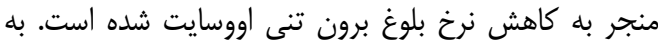

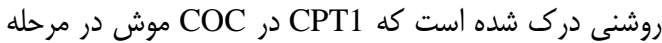

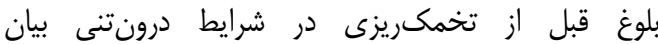

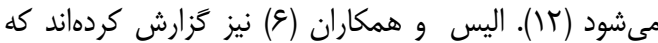

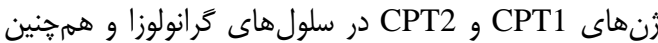
بخشهاى ديگر تخمدانى مانند جسم زرد، فوليكول،

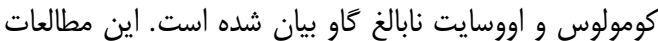

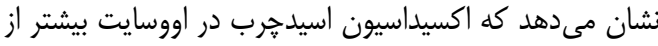

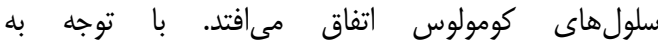

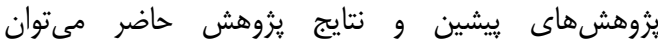

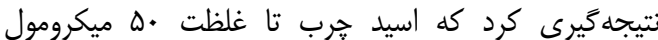

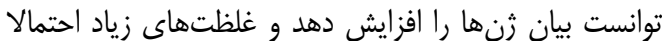

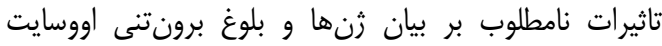

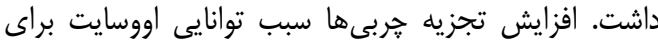

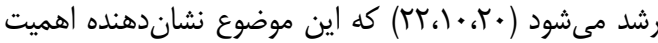

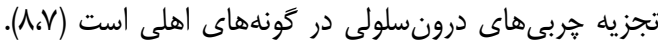

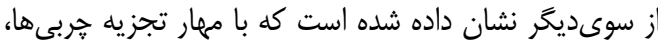

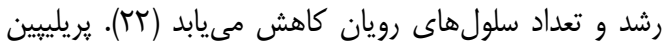

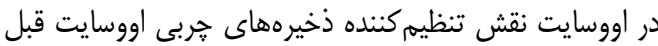

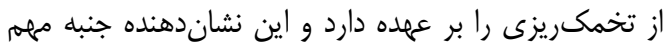

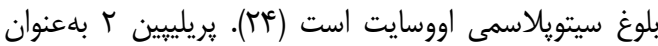

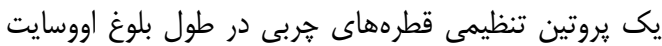

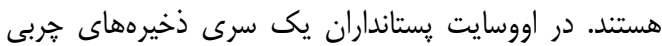

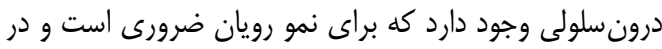

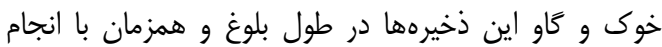

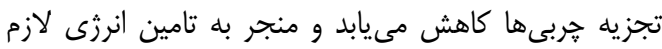

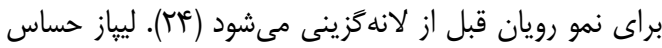

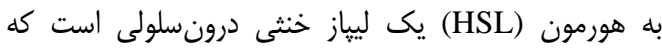

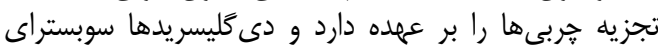

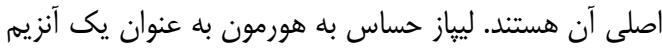

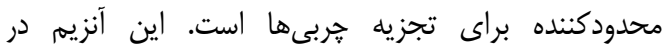

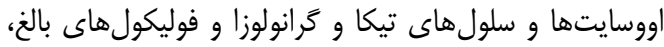

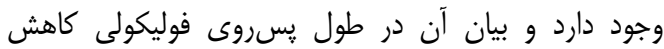

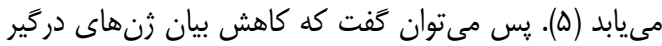

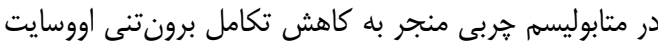

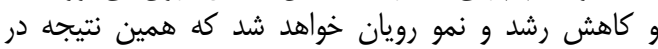

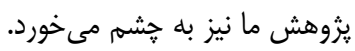

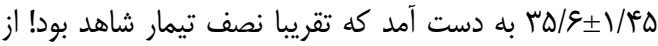

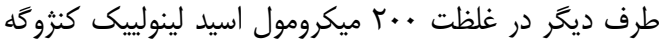

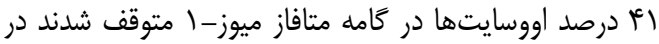

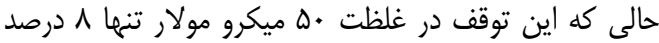

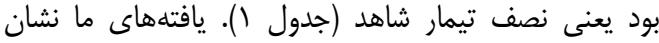

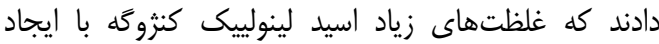

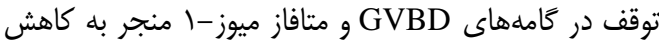

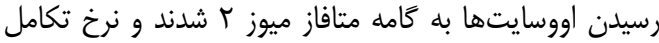

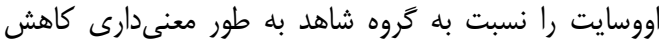

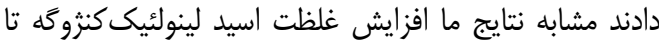

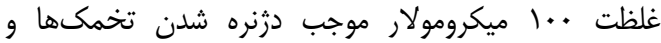

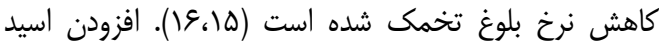

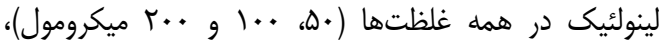

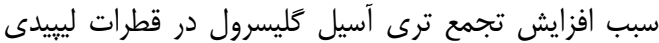

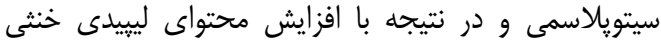

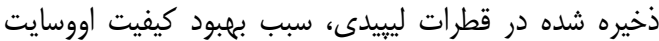

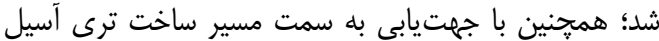
كليسرول و افزايش درجهى غيراشباع فسفوليييدهاى غشاءئ ترى

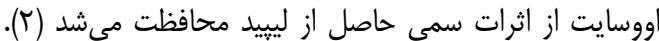

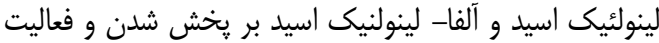

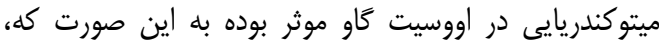

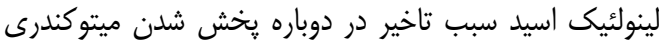

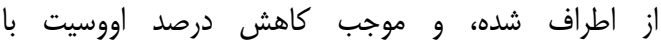

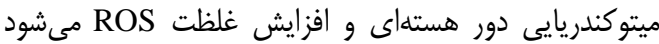

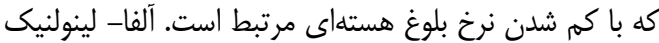

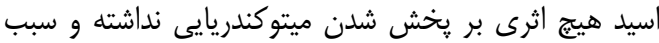

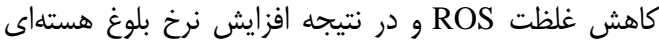

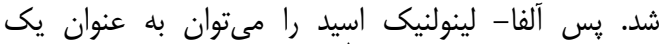

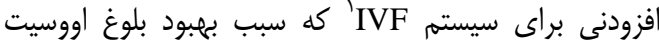

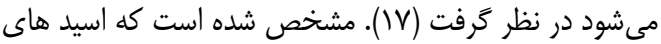

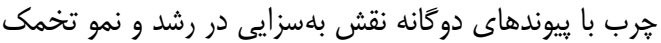

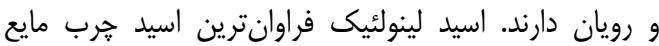

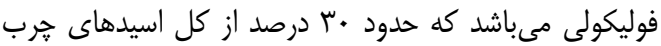

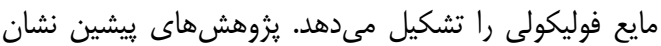

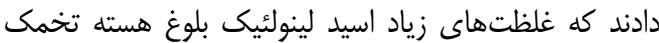

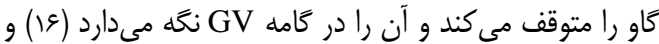

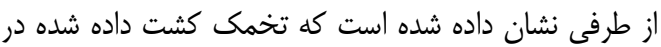

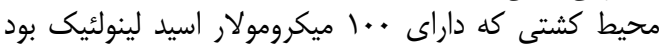

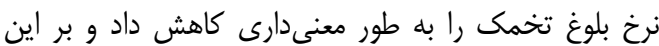

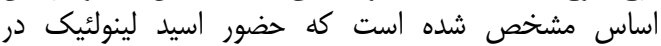

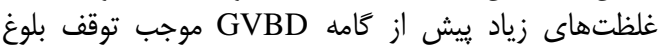

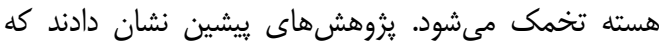

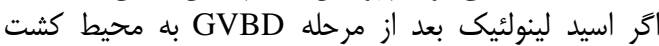

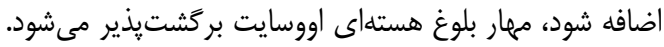

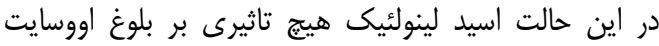

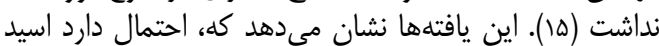

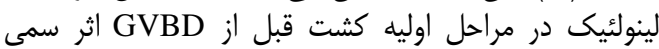

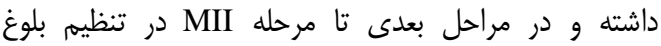

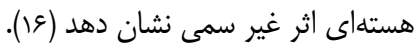


1. Aardema, H., P.A.L. M. Vos, F. Lolicato, B.A.J. Roelen, H.M. Knijn, A.B. Vaandrager, J.B. Helms, and B.M. Gadella. 2011. Oleic acid prevents detrimental effects of saturated fatty acids on bovine oocyte developmental competence. Biology Of Reproduction, 85: 62-69.

2. Carro, M., J. Buschiazzo, G.L. Ríos, G.M. Oresti and R.H. Alberio. 2013. Linoleic acid stimulates neutral lipid accumulation in lipid droplets of maturing bovine oocytes. Theriogenology, 79: 687-694.

3. Cetica, P., L. Pintos, G. Dalvit and M. Beconi and M. 2002. Activity of key enzymes involved in glucose and triglyceride catabolism during bovine oocyte maturation in vitro. Reproduction, 124: 675681.

4. Dunning, K.R., K. Cashman, D.L. Russell, J.G. Thompson, R.J. Norman and R.L. Robker. 2010. Beta oxidation is essential for mouse oocyte developmental competence. Biology Of Reproduction, 83: 909-918.

5. Dunning, K.R., D.L. Russell and R.L. Robker. 2014. Lipids and oocyte developmental competence: the role of fatty acids and beta-oxidation. Reproduction, 148: 15-27.

6. Elis, S., A. Desmarchais, V. Maillard, S. Uzbekova, Ph. Monget and J. Dupont. 2015. Cell proliferation and progesterone synthesis depend on lipid metabolism in bovine granulosa cells. Theriogenology, 83: 840-853.

7. Ferguson, E.M. and H.J. Leese. 1999. Triglyceride content of bovine oocytes and early embryos. Reproduction, 116, 373-378.

8. Ferguson, E.M. and H.J. Leese. 2006. A potential role for tryglyceride as an energy sources during bovine oocyte maturation and early embryo development. Molecular Reproduction and Development, 73: 1195-1201.

9. Leese, H.J. and E.M. Ferguson. 1999. Embryo metabolism. In: Jansen R, Mortimer D(eds). Towards Reproductive Certainty. New York: Parthenon, 360-366.

10. Ferreira, E.M., A.A. Vireque, P.R. Adona, F.V. Meirelles, R.A. Ferriani and P.A.A.S Navarro. 2009. Cytoplasmic maturation of bovine oocytes: structural and biochemical modifications and acquisition of developmental competence. Theriogenology, 71: 836-848.

11. Flynn, T.J. and N. Hillman. 1980. The metabolism of exogenous fatty acids by preimplantation mouse embryos developing in vitro. Journal of embryology and experimental morphology, 56: 157-168.

12. Gentile, L., M. Monti, V. Sebastiano, V. Merico, R. Nicolai, M. Calvani, S. Garagna, C.A. Redi and M. Zuccotti. 2004. Single-cell quantitative RT-PCR analysis of Cpt1b and Cpt2 gene expression in mouse antral oocytes and in preimplantation embryos. Cytogenetic and Genome Research, 105: 215 221.

13. Lapa, M., C.C. Marques, S.P. Alves, M.I. Vasques, M.C. Baptista, I. Carvalhais, M.S. Pereira, A.E.M. Horta1, R.J.B. Bessa and R.M. Pereira. 2011. Effect of trans-10 cis-12 conjugated linoleic acid on Bovine Oocyte Competence and Fatty Acid Composition. Reproduction in Domestic Animals, 46: 904-910.

14. Leroy, J.L.M.R., R.G. Sturmey, V. Van Hoeck, J. De Bie, P.J. McKeegan and P.E.J. Bols. 2013. Dietary lipid supplementation on cow reproductive performance and oocyte and embryo viability: a real benefit? Animal reproduction, 3: 258-267.

15. Marei, W.F., D.C. Wathes and A.A. Fouladi-Nashta. 2009. The Effect of linolenic acid on bovine oocyte maturation and development. Biology Of Reproduction. 81: 1064-1072.

16. Marei, W.F., D.C. Wathes and A.A. Fouladi-Nashta. 2010. Impact of linoleic acid on bovine oocyte maturation and embryo development. Reproduction, 139: 979-988.

17. Marei, W.F., D.C. Wathes and A.A. Fouladi-Nashta. 2012. Differential effects of linoleic and alphalinolenic fatty acids on spatial and temporal mitochondrial distribution and activity in bovine oocytes. Reproduction. Fertility and Development, 24: 679-690. 
18. McEvoy, T.G., G.D. Coull, P.J. Broadbent, J.S. Hutchinson and B.K. Speake. 2000. Fatty acid composition of lipids in immature cattle, pig and sheep oocytes with intact zona pellucida. Journal of Reproduction and Fertility, 118: 163-170.

19. Pereira, R.M., M.C. Baptista, M.I. Vasques, A.E.M. Horta, P.V. Portugal, R.J.B. Bessa, E. Chagas and J. Silva. 2007. Cryosurvival of bovine blastocysts is enhanced by culture with trans-10 cis-12 conjugated linoleic acid (10t,12c CLA). Animal Reproduction Science, 98: 293-301.

20. Sirard, M.A., F. Richard, P. Blondin and C. Robert. 2006. Contribution of the oocyte to embryo quality. Theriogenology, 65: 126-136.

21. Sturmey, R.G., P.J. O'Toole and H.J. Leese. 2006. Fluorescence resonance energy transfer analysis of mitochondrial lipid association in the porcine oocyte. Reproduction, 132: 829-837.

22. Sturmey, R.G. and H.J. Leese. 2003. Energy metabolism in pig oocytes and early embryos. Reproduction, 126: 197-204.

23. Sturmey, R.G., A. Reis, H.J. Leese and T.G. McEvoy. 2009. Role of fatty acids in enery provision during oocyte maturation and early embryo development. Reproduction of Domestic Animals, 44: 5058.

24. Yang, X., K.R. Dunning, L.L.Wu, T.R. YHickey, R.J. Norman, D.L. Russell, X. Liang and R.L. Robker. 2010. Identification of Perilipin-2 as a lipid droplet protein regulated in oocytes during maturation. Reproduction, Fertility and Development, 22: 1262-1271.

25. Zeron, Y., A. Ocheretny, O. Kedar, A. Borochov, D. Sklan and A. Arav. 2001. Seasonal changes in bovine fertility: relation to developmental competence of oocytes, membrane properties and fatty acid composition of follicles. Reproduction, 121: 447-454. 


\title{
Effect of Conjugated Linoleic Acid On Gene Expressions Involved in Lipid Metabolism in In Vitro Matured Sheep Oocytes
}

\author{
Hamid Deldar ${ }^{1}$, Zarbakht Ansari Pirsaraei ${ }^{2}$ and Maryam Royan ${ }^{3}$ \\ 1- Associate Professor, Department of Animal Science, Sari Agricultural Sciences and Naural Sciences University \\ (Corresponding Author: h.deldar@sanru.ac.ir) \\ 2- Associate Professor, Department of Animal Science, Sari Agricultural Sciences and Naural Sciences University \\ 3- North Region Branch, Agricultural Biotechnology Research Institute of Iran (ABRII), Agricultural Research, \\ Education and Extension Organization (AREEO), Rasht, IRAN \\ Received: April 10, $2018 \quad$ Accepted: May 16, 2018
}

\begin{abstract}
The aim of this study was to investigate the effect of different concentrations of conjugated linoleic acid (CLA) on in vitro maturation (IVM) and lipid metabolism-related genes in oocytes. In vitro maturation of oocyte was performed in the presence of control (0.0), 50, 100, and 200 $\mu \mathrm{M}$ CLA. Nuclear status and mRNA abundance of selected genes were evaluated following 24 $\mathrm{h}$ of IVM. Following the IVM, fertilization and embryo culture were carried out in all groups and embryonic development was examined. The addition of $50 \mu \mathrm{M}$ CLA to the maturation media yielded a higher number of oocytes at MII stage in comparison to the control group (85.3 \pm 1.94 versus $68.0 \pm 3.04)$. Higher concentration of CLA $(200 \mu \mathrm{M})$ was significantly decreased $(P<0.05)$ the maturation rate of oocyte $(35.6 \pm 1.45)$. Cleavage, and blastocyte rate were higher in the $50 \mu \mathrm{M}$ CLA group in comparison to the control one. Also, the $200 \mu \mathrm{M}$ CLA were significantly decreased the cleavage and blastocyst rate (32.87 and 13.69, respectively). The relative expression of perilipin-2 and hormone sensitive lipase were significantly increased following the addition of $50 \mu \mathrm{M}$ CLA to the maturation media. Higher concentrations of CLA were significantly dropped the mRNA abundance of both perilipin-2 and hormone sensitive lipase gene in the oocyte. The CPT-1 mRNA abundance was not influenced by the addition of different concentrations of CLA to the maturation media. It seems that the $50 \mu \mathrm{M}$ CLA yielded a higher number of embryo in comparison to the higher concentration of CLA and excessive concentrations of CLA had undesirable effect on oocyte maturation and embryo development of ovine oocyte.
\end{abstract}

Keywords: Conjugated linoleic acid, in vitro maturation, Embryo development, Lipid metabolism 